\title{
Chapter 3 \\ The Promise and Neglect of Follow-up \\ Care in Obstetric Fistula Treatment \\ in Uganda
}

\author{
Bonnie Ruder and Alice Emasu
}

\section{Introduction}

In the calm before the afternoon storm, the regional referral hospital in central Uganda was uncharacteristically quiet. The parking lot and hospital grounds were almost completely empty. In the maternity ward, only a few postpartum mothers and family members occupied the otherwise vacant space. Missing were the long lines of women waiting for care - the norm in maternity wards throughout Ugandaalong with the chaotic bustle of the hospital grounds that I had grown accustomed to. It was four days into a national doctors' strike, in which doctors were protesting appallingly low wages and a chronically underfunded healthcare system. The news of the strike had spread throughout Uganda, persuading everyone except those with dire emergencies to stay home.

For our study team, the strike meant that the surgeon scheduled to conduct fistula screenings was called away to handle the latest emergency. Several hours passed while I waited with a fistula counselor and translator who works for a local NGO, a nurse, and 12 potential study participants who were waiting to be screened. The study was designed to investigate the etiology, severity, and quality of life for women experiencing persistent residual incontinence after surgical closure of an obstetric fistula. All of these women had undergone surgery for obstetric fistula, yet they were still leaking urine. The women sat next to each other on a long wooden bench and quickly bonded over their shared affliction-their incontinence and the suffering it brought.

\footnotetext{
B. Ruder $(\bowtie)$

Terrewode Women's Fund, Soroti, Uganda

e-mail: bruder@terrewodewomensfund.org
}

\section{A. Emasu \\ TERREWODE, Soroti, Uganda \\ e-mail: alice@terrewode.com}


Many hours later, the doctor appeared ready to screen the women. Fistula screening involves a relatively simple test in which a catheter is inserted into the urethra and the bladder is backfilled with a blue dye that is used to diagnose vesico-vaginal fistulas and stress incontinence. Ten of the 12 women passed the screening; this meant their fistula was closed yet they were still leaking urine, thus they qualified for participation in the study. The two women who did not qualify were informed that their fistulas were still open and that they may need another surgery to close their fistula. The counselor and surgeon met with each woman individually to explain their findings and discuss treatment options. Of the ten women who qualified for the study, several were initially dry upon discharge from their fistula surgery yet started to leak urine again days or months later. Other women started leaking immediately following surgery; however, this screening was the first time a healthcare worker had explained that they were experiencing residual incontinence rather than a failed closure. In both circumstances, the women had either not received follow-up care or returned for follow-up but were still confused about their prospects for treatment. All of the women were clear, however, that they desperately wanted to be cured from the continuous leaking.

\section{- Fieldnotes, first author, October 2017}

This chapter presents findings from a community-led project with a Ugandan nongovernmental organization (NGO) that was designed to investigate both the clinical profile and qualitative experiences of women with post-repair incontinence. The chapter draws on ethnographic fieldwork and narratives collected through open-ended interviews with fistula surgeons and women suffering from residual incontinence. Based on the emergent themes, we conducted a desk review of international and Ugandan fistula policies and guidelines, tracing fistula policy in Uganda to understand the current approach to follow-up care after fistula treatment. We discovered vague fistula policies, absent guidelines, and unfunded mandates, especially in relation to follow-up care. Ultimately, we argue that an inadequate treatment model that neglects follow-up care exists. We argue that this neglect can be traced to a donor-funded delivery model that fails to prioritize, or fund, follow-up care as an essential component of fistula treatment. As a result, poor outcomes are underreported and women who experience poor outcomes are largely erased from the fistula narrative. This erasure shapes treatment possibilities and research priorities, leaving out women still in need of additional treatment.

\section{Obstetric Fistula in Uganda}

International discussions about maternal health in low-income countries tend to focus on maternal deaths. However, these deaths are only the tip of the iceberg in terms of the health effects of the poor availability and quality of maternity services (Paxton \& Wardlaw, 2011; Storeng et al., 2012). Closely entwined with maternal mortality and propelled by the same health system deficiencies are maternal 
morbidities, injuries women suffer during pregnancy, childbirth, or postpartum. Assumed to be directly or indirectly related to difficult obstetric events, these morbidities/disabilities include conditions such as uterine prolapse, stress incontinence, hypertension, hemorrhoids, perineal tears, urinary tract infections, severe anemia, depression, fistula, and ectopic pregnancy (Koblinsky et al., 2012). The World Health Organization (WHO) estimates that for every maternal death, 30-50\% of women who survive suffer humiliating or disabling injuries (WHO, 2015).

Beyond the acute impact of obstetric complications and the potential for future morbidities and disabilities, maternal morbidities have secondary consequences for women and their families. These consequences extend the meaning of loss beyond quantitative indicators such as the maternal mortality ratio or Disability-Adjusted Life Years (DALYs). For instance, studies from Burkina Faso tell of consequences that can span over several years and include catastrophic health expenditure, severe and persistent illness - including increased risk of all cause and pregnancy related death in the ensuing 4 years and mental health problems - stigmatization, violence, isolation and divorce, and loss of family stability and community status (Filippi et al., 2007; Storeng et al., 2010).

The most severe of maternal morbidities is an obstetric fistula. Obstetric fistula is a traumatic childbirth injury caused most often by prolonged, obstructed labor, due to either fetal malpresentation or more commonly, cephalo-pelvic disproportion-where the fetus's head is unable to fit through a woman's pelvis during labor. During the prolonged labor, the continuous pressure of the fetal head on the bladder or rectal tissues causes diminished blood flow. Eventually the tissue becomes necrotic and sloughs off, leaving a small hole, or fistula, between the woman's vaginal walls and the bladder and/or rectum. The result is uncontrollable leakage of urine and/or feces. In most cases of obstetric fistula, the fetus dies. The pressure exerted by the fetus during prolonged labor can also damage nearby organs and nerves. The multisystem injuries, described as the "obstructed labor injury complex," include renal failure, secondary infertility, foot drop from nerve injury, vaginal stenosis, and chronic skin maceration ${ }^{1}$ (Arrowsmith et al., 1996).

The social and emotional consequences of living with fistula are also extreme, as the odor from continuous incontinence often leads to discrimination and social shaming from friends, family, and community members. Women with fistula commonly report feeling embarrassment, loneliness, worry, sadness, disappointment, and anger (Mselle et al., 2011; Pope et al., 2011; Weston et al., 2011). Women with fistula also report serious mental health issues, including severe depression and suicidal tendencies (Browning et al., 2007; Khisa et al., 2017). Divorce, separation, and marital discord are common (Ahmed \& Holtz, 2007; Barageine et al., 2015;

\footnotetext{
${ }^{1}$ Foot drop is caused from peroneal-nerve compression during labor and results in weakness or paralysis of the forefoot. Women with foot drop experience difficulty lifting their foot and thus have limited mobility. Chronic skin maceration is the breakdown of skin on the labia and inner thighs which occurs due to the prolonged exposure to moisture. Vaginal stenosis is the narrowing and loss of flexibility in the vagina, resulting in painful intercourse.
} 
Mwini-Nyaledzigbor et al., 2013), though not universal, experiences for women with fistula.

Today, obstetric fistulas are extremely rare in high-income countries. However, in low-resource countries with underfunded and poorly provisioned healthcare systems and limited access to quality emergency obstetric care, especially caesarean delivery, fistula remains a public health problem. The WHO (2018) estimates that in sub-Saharan Africa and Asia more than two million women live with untreated fistula, yet reliable prevalence data on obstetric fistula is unavailable. Prevalence of obstetric fistula is difficult to determine in part because women are often reluctant to reveal their symptoms, and due to the fact that true prevalence can only be confirmed with clinical examination, rendering large community-based prevalence studies cost prohibitive.

The primary intervention for women with obstetric fistula is surgical treatment, with reported success rates between $80 \%$ and $97 \%$ (Barone et al., 2012; Delamou et al., 2016; Ouedraogo et al., 2018). Following treatment, women typically report improvements in their social life and relationships, income-earning opportunities, and overall quality of life (Drew et al., 2016; El Ayadi et al., 2019; Pope et al., 2011).

However, access to fistula treatment in Uganda, similar to other low-resource countries with high fistula burdens, is severely limited. In Uganda, an estimated 114,000 women currently suffer from fistula with an additional 1,900 new cases annually (UBOS, 2018), yet due to the chronically underfunded and underprovisioned healthcare system, treatment capacity is only estimated at 1,670 cases per year (UBOS, 2018). Health facilities are plagued by a lack of infrastructure and equipment, essential drugs, and even basic supplies. Uganda also struggles with severe health worker shortages and an inadequate distribution of health workers, with the majority of health workers located in urban areas despite a predominantly rural population. Compounding this are the low wages - the major complaint during the national doctors' strike-poor morale, inadequate training, and low-staff competence, all of which result in poor quality of care, which recent studies have shown is even more pronounced in rural health facilities (Kruk et al., 2016).

In response to these healthcare deficiencies, Uganda turned to fistula surgical camps, which were initially promoted by international development agencies, including UNFPA, the African Medical and Research Foundation (AMREF), and the WHO, in Uganda and throughout sub-Saharan Africa as a short-term solution to deal with the huge backlog of patients while simultaneously building surgical capacity in countries most affected by obstetric fistula (Ramsey et al., 2007; Hancock \& Collie, 2004). The two-week surgical camps are usually hosted at public hospitals and are offered several times a year at different locations throughout the country with either a foreign surgeon and/or one or more Ugandan surgeons. Promoted via radio announcements, it is common for 50 or more women to receive treatment during this two-week period, though sometimes many more women turn up, resulting in overcrowding, long surgical days that stress support staff at the hospital, and women being turned away when time or funding runs out (Ruder et al., 2018). Routine treatment at referral hospitals has been introduced in Uganda, yet is still not available in most areas of the country. 
While the majority of women who have undergone a successful surgical repair will remain continent, as many as $16-55 \%$ may experience residual incontinence post-fistula closure in the form of stress incontinence, urge incontinence, and increased urinary urgency and frequency (Murray et al., 2002; Wall et al., 2004). Clinical studies have reported risk factors for developing residual incontinence, which include urethral involvement, reduction in the size of the bladder, the size and location of the fistula, and the extent and severity of vaginal scarring (Browning, 2006; Goh et al., 2008). Studies that describe women's experiences with residual incontinence reveal that they experience a lower quality of life, continued mental health concerns, stigma, and lower levels of social support than women who are successfully repaired (Browning, 2012; Donnelly et al., 2015; El Ayadi et al., 2019; Pope et al., 2011; Wilson et al., 2016).

Despite calls for more research, residual incontinence remains an underexplored and poorly tracked outcome of fistula treatment. In a recent review of current challenges in the field of obstetric fistula, Rane et al. (2020) cite residual incontinence as one of the most pressing problems - one that is not well understood and for which there are no clear treatment solutions. This is due in part to the fact that fistula outcomes and surgical success rates are based on a clinical assessment conventionally conducted at discharge, typically 14 days after the surgery. However, repair breakdowns and residual incontinence may occur up to six months or more after discharge, invalidating these results. Furthermore, for years, treatment success has been narrowly defined solely by the closure of the fistula defect. Thus, "success" rates may fail to provide an accurate account of whether the woman is actually dry following the surgery (versus closed fistula with residual incontinence), and they fail to capture long-term continence of the patient. Despite a call more than a decade ago for data on surgical outcomes based on both closure and continence, the literature on fistula surgery remains plagued by the absence of clear definitions of "success" and "failure" following surgical treatment (Harrison et al., 2015; Wall \& Arrowsmith, 2007).

Quality follow-up care for patients could provide a more accurate assessment of long-term continence results, yet the majority of fistula treatment programs lack a systematic follow-up protocol (Morren et al., 2016). Additionally, few studies document follow-up care post-surgery, which many argue is too expensive and impractical to be implemented given the local context — in which women often travel long distances from remote areas to receive treatment (Murray et al., 2002; Lewis \& De Bernis, 2006). While the number of studies reporting on long-term follow-up is growing, the vast majority report fistula outcomes upon hospital discharge. To our knowledge, this study is the first to report both women's and surgeons' experiences with follow-up care, and to trace follow-up guidelines in fistula policy.

When speaking of policy, we draw on Gilson (2012) who notes that health policy refers to the formal written documents, rules and guidelines created to promote and improve health. Policy making is often described as a multistep process, two essential features of which are central here: (1) evaluation to assess effectiveness and any unforeseen outcomes; and (2) revision to make necessary adjustments to better achieve desired outcomes (Buse et al., 2005). This study reveals how these essential 
steps are unattended to in fistula policy - to the detriment of women with residual incontinence.

\section{Methods}

This chapter draws on qualitative results from a community-led project with a Ugandan NGO that provides fistula treatment and reintegration services, including psychosocial counseling, safe motherhood and sexual health and reproductive rights education, and income generating skills training. This study was part of the first author's larger dissertation study that utilized a mixed-method study design to investigate the experiences of women suffering from residual incontinence postfistula closure $(n=36)$. We triangulated the findings with semi-structured, openended interviews with fistula surgeons-both Ugandan and international surgeons - who work or have worked in Uganda $(n=12){ }^{2}$

Participants were recruited using purposive sampling (Bernard, 2006). Data collection took place between October 2017 and September 2018 in eastern and central Uganda, regions chosen due to the high volume of fistula treatment and the established community presence of the Ugandan NGO we collaborated with. Semistructured interviews lasted an average of one hour and were conducted at the NGO's office or at a local conference room reserved for privacy. The first author, a medical anthropologist and midwife, conducted the interviews with the assistance of local female translators who are also trained fistula counselors, familiar with the sensitive nature of fistula. Interviews were conducted in the participant's native language, Ateso, Kumam, or Lugandan, and audio recorded with their permission, translated into English, and transcribed verbatim. Interviews with fistula surgeons lasted an average of one hour and were conducted in English at a location convenient to the surgeon or via Skype if the surgeon was out of the country.

In order to situate the emergent themes of the findings, we conducted a desk review focused on the broader field of obstetric fistula policy. We searched unpublished ("grey") literature (Google, organizational websites, Ugandan Ministry of Health $(\mathrm{MoH})$ website). We were also able to locate hard copies of Ugandan $\mathrm{MoH}$ policy documents that are not available online, looking specifically for policy guidelines for follow-up care post-fistula treatment.

Collectively, we also drew on participant observation from extensive fieldwork on fistula research projects in Uganda, participation in Ugandan $\mathrm{MoH}$ and Fistula Technical Working Group (FTWG) meetings, and attendance at international global health and obstetric fistula conferences in order to situate fistula policy.

Transcripts were coded and sorted into emerging themes using Nvivo 12.2. Analysis was conducted using modified grounded theory, an iterative method that requires a close interaction with the data in order to allow emic themes to emerge,

\footnotetext{
${ }^{2}$ In order to respect confidentially, no identifying details on the surgeons are provided.
} 
followed by theoretical construction based on the emergent themes (Charmaz, 2006). Informed consent was obtained from all participants who enrolled in the study, with signature or thumbprint. The study protocol was approved by the Institutional Review Board at Oregon Health Sciences University, Oregon State University, Makerere University College of Health Sciences, and the Uganda National Council for Science and Technology.

\section{Obstetric Fistula Emerges as an International Priority}

Once virtually unknown, obstetric fistula emerged as an international priority in the early 2000s, following two decades of focus on maternal mortality and safe motherhood. The United Nations Population Fund (UNFPA) was the first to champion the cause on the international stage with their "Campaign to End Fistula" in 2003. They drew in dozens of partners to the campaign, including large funders such as the Gates Foundation, corporate sponsors such as Virgin Atlantic, and celebrity spokespersons such as British-American singer-songwriter Natalie Imbruglia (UNFPA, 2008).

In the early years, the campaign worked with countries to produce baseline needs assessments, and then offered financial and technical assistance to ministries of health to create new national guidelines and strategies to tackle fistula, and to establish national technical working groups (UNFPA \& EngenderHealth, 2003; UNFPA, 2008). UNFPA continues to promote and raise funds for the campaign, including serving as the secretariat of the International Fistula Working Group, which coordinates global efforts to eliminate fistula (UNFPA, 2008). The Campaign to End Fistula has led to numerous UN resolutions on supporting efforts to end obstetric fistula $(2007,2010$, and 2012) and resolutions on the intensification of efforts to end obstetric fistula $(2014,2016,2018$ \& 2020) including the development of a global road map within a decade (UN, 2018). Their goal is to end fistula by 2030 (Anastasi et al., 2017).

\section{Fistula Policy in Uganda}

Obstetric fistula policy has been determined in large part over the years by international development agencies and funding organizations, such as international nongovernmental organizations (INGOs). These agencies and organizations have provided millions of dollars in resources and focused international attention on the issue of obstetric fistula. In many cases, they have provided the only funding for fistula treatment in low-resource countries. These organizations have thus played a critical role in assisting national ministries of health in formulating individual country-level policies regarding fistula treatment. 
This is certainly the case in Uganda, where for years international development agencies have worked alongside the Ugandan $\mathrm{MoH}$ to formulate a national fistula policy. While fistula treatment started in Uganda in the 1990s with a few dedicated international surgeons providing fistula surgery and training Ugandan surgeons, it was not until the 2000s that Uganda began to formulate fistula guidelines. Uganda was included in UNFPA's initial nine country fistula assessment, conducted in 2002, the year prior to the UNFPA kick-off of their Campaign to End Fistula (UNFPA \& EngenderHealth, 2003). Also in 2002, with funding and assistance from the UNFPA, the Ugandan MoH founded the Fistula Technical Working Group (FTWG). The FTWG is made up of a diverse group of stakeholders including $\mathrm{MoH}$ representatives, Ugandan fistula surgeons, international development partners such as UNFPA, the African Medical and Research Foundation (AMREF), EngenderHealth, and the WHO, and Ugandan civil society and NGOs (Creanga et al., 2008). The purpose of the FTWG is to coordinate fistula stakeholder activities and integrate fistula services into the maternal healthcare system. Before the group was established, there was no national oversight or coordination for fistula prevention and treatment in Uganda. The group has been instrumental in creating Uganda's fistula policies, such as the Minimum Package for Conducting Fistula Camps (Ministry of Health, 2014) and the National Obstetric Fistula Strategy (Ministry of Health, 2011).

Aside from establishing standards, guidelines, and protocols to guide the provision of fistula services and "integrating fistula services into the Ugandan health system" the group reports its achievements as "building an information base for obstetric fistula, to better plan for and manage prevention, treatment, and reintegration services," which includes increasing the number of fistula questions on the Ugandan Demographic and Health Survey (a nationally representative household survey conducted every 5 years) from one to three, and beginning the process of integrating key fistula indicators into routine administrative data so that health facilities can regularly report on fistula cases (Fistula Care, 2013, p. 2). However, the group is plagued by a lack of resources and insufficient implementation support from the $\mathrm{MoH}$, and despite their achievements, fistula strategies in Uganda remain poorly implemented and disjointed with specific deficits in "data systems, capacity building, clinical standards, and support by partners" (Ministry of Health, 2011, p. 7).

\section{In Search of Follow-up Protocols}

Surprisingly, an exhaustive search for follow-up care protocols revealed that followup care is rarely mentioned in international or Ugandan guidelines. In fact, according to the WHO, "once healed, [women] return to far-off villages in remote regions making follow-up impossible" (Lewis \& De Bernis, 2006, p. 71). When follow-up care is included, instructions are often vague, such as in "The prevention and management of obstetric fistula: A curriculum for nurses and midwives," which states that follow-up appointments should occur within 3 months and that nurses and midwives are to "ensure that the client has the necessary means and transportation to 
come back for the visit" (emphasis added) (ECSA-HC, 2012, p. 220). Exactly how nurses and midwives are to "ensure" this unfunded mandate is not discussed.

In Uganda's national guidelines, follow-up care often falls under reintegration rather than clinical protocols and is relegated to NGOs and community organizations. For example, Uganda's Minimum Package for Conducting Fistula Camps (2014) states "NGOs can follow-up the patients at home at one, three and six months and one year after her return to her village" (p. 13). In Uganda's National Training Guidelines (2012b) the responsibility for follow-up care is shifted to village health teams, counselors, and community representatives. No mention of reporting or referral mechanisms for follow-up care is made. As further evidence of the absence, the MoH Fistula Reporting Data Forms (2012), developed with development partners EngenderHealth, UNFPA, and AMREF, fail to include follow-up care or outcomes among the reportable data fields.

Even a relatively recent report from Fistula Care and EngenderHealth (2013, p. 5), which celebrated Ugandan achievements and expected progress, and stated: "Health care providers must follow consistent approaches for prevention and treatment that are grounded in current medical knowledge and evidence," failed to include follow-up care in their guidelines and protocols, despite outlining service standards and the importance of quality of care.

The lack of specificity around follow-up care in international and national guidelines comes despite the fact that the International Federation of Gynecologists and Obstetricians (FIGO) Global Competency-Based Fistula Surgery Training Manual from 2011 included specific competencies on follow-up care (FIGO, 2011). Likewise, the UN began calling for "careful" follow-up by December 2012, and has continued to do so in every subsequent declaration on obstetric fistula, including the 2016 resolution which stated: "governments of countries affected by fistula should designate obstetric fistula as a nationally notifiable condition, triggering immediate reporting, tracking and follow-up" (UN, 2016, p. 11).

We have observed that the lack of attention to follow-up care in policy documents is also reflected in conference proceedings and meetings. For instance, the biannual International Conference of Obstetric Fistula Surgeons, which began in 2007, has yet to highlight the issue. One notable exception was during the 2014 conference in Kampala, Uganda when a well-respected surgeon took the floor to express his concerns about the lack of follow-up care and demanded to know "Who is paying for follow-up?" (October 2014, fieldnotes, first author). Similarly, at national meetings in Uganda, such as FTWG meetings, follow-up is seldom prioritized, even when guidelines and quality of care strategies are discussed. In the following sections, we share the results from semi-structured, open-ended interviews with women suffering from residual incontinence post-fistula closure and fistula surgeons. 


\section{Women's Experiences with Fistula Follow-up Care}

This study offers a unique understanding of barriers to follow-up care because the women in our study were still leaking after their surgery and thus, were especially motivated to attend follow-up appointments. Women often returned multiple times for follow-up care and the majority, 83\%, eventually received additional surgery. This corresponds with other studies on residual incontinence. For example, Browning and Menber (2008) found that women with residual incontinence symptoms were twice as likely to return for follow-up than women free of symptoms. At first glance, the high rate of follow-up in this study appears to be a success; however, the in-depth interviews present a more troubling picture of women's experience with follow-up care, which is closely tied with their experience of receiving an unclear diagnosis.

\section{Unclear Diagnosis}

The majority of the women who participated in this study were unaware that their fistula was actually closed and still spoke of themselves as having a fistula, as seen in the opening vignette. Here a 45-year-old woman who had eight prior surgeries explains, "When I was discharged on my last treatment, the doctors did not tell me that all my fistulas had been closed. Instead, I only knew it yesterday, when I was screened at the hospital."

Another woman who has had a total of five surgeries shared,

The last surgery, the doctors did not tell me anything about why I was still leaking. In fact, I was so disappointed. Because after leaving [the regional hospital] and going to Mulago [the national referral hospital], I was thinking that I would come back when I was dry. I got so traumatised. Then after that, they even didn't tell me what I should do.

This finding corroborates our previous research findings, which show that women receive minimal information from medical personnel about their initial diagnosis of fistula (Ruder et al., 2018). The lack of clarity regarding their diagnosis was also a source of distress for the women in this study, and most reported that they were not given medical advice specific to residual incontinence. Very few women understood their fistula was closed and that the cause of the leaking was residual incontinence. Instead of clear medical advice and a diagnosis, the majority were told that maybe something could be done for them in the future; they should go home and wait for an announcement for another camp. This poor understanding of their medical condition led women to repeatedly seek out both follow-up care and additional treatment.

Surgeons who participated in this study did take the time to explain the cause of the incontinence, using hand gestures and easy-to-understand descriptions such as "the fistula is closed, but the 'brakes' which hold in your urine have been damaged and are not working correctly." Women quickly understood this diagnosis was 
different from a failed fistula repair. This simple explanation seemed to alleviate much of the suffering that resulted from the uncertainty they reported after repeated surgeries without improvement, as this woman shared after her screening: "I'm glad the doctor told me I don't have a hole, it's now the brakes, the muscles that are weak."

\section{Barriers to Follow-up}

Both women participants and surgeons identified multiple barriers women experience in receiving follow-up care. According to women, foremost amongst these were transportation costs. As this woman describes clearly: "I did not go back for review because I did not have any money for transport to the hospital." While women are often reimbursed for transportation costs when they travel for a fistula camp, transportation costs for follow-up appointments are not typically reimbursed by donors. For woman with residual incontinence, they have already raised funds needed for the surgery. ${ }^{3}$ Now, suffering from what they think of as a "failed surgery," they must again raise funds to return for additional medical care. Additionally, to attend both the fistula camp and follow-up appointments, women often travel long distances on crowded public buses where it is difficult to conceal their incontinence, thus returning six weeks later is a significant burden.

Tenacity and perseverance were common themes in this study however, as women overcame hardship and repeatedly sought additional care. Unfortunately, their stories also highlight the dysfunction with follow-up care in Uganda. A woman who suffered with residual incontinence for six years shared:

So, the last time I was in hospital for surgery, the doctors advised me to do [Kegel] exercise for one month. He told me in the morning I should do it ten times, the holding of the muscles, then in the evening ten times. He told me to call them after one month, but when I called the sister [nurse], the contact number they had given me, she never picked up her phone.

Kegel exercises are a standard treatment for some forms of residual incontinence, yet in many cases of severe residual incontinence post-fistula closure, prove insufficient as treatment.

Many women were simply told to return home and wait for a call to return for additional treatment, which despite their extreme patience-sometimes after waiting years-never came. Others described poor continuity of care; these women returned to the hospital where they were treated during a fistula camp, only to find that the doctors who conducted their surgery were no longer there. Instead they found junior doctors or clinical officers who were unable to explain to the women why they were still leaking or what course of action they should take. These women were frustrated and often lost hope because of this experience.

\footnotetext{
${ }^{3}$ Even when surgical costs are covered by a donor, women typically need money for food and upkeep at the hospital, and for the family member who has accompanied them.
} 


\section{Surgeon's Perspectives on Follow-up}

According to surgeons who participated in this study, the standard of practice in Uganda is to recommend a follow-up appointment six weeks post-surgery, though as stated above, unlike fistula treatment, follow-up is not a donor-funded activity. Surgeons report that they routinely ask women treated for fistula to return for follow-up appointments; however, most do not return. They pointed to several structural barriers that impede follow-up visits, including transportation costs, long distances to the hospital, and difficulty missing work or leaving children to attend the appointment. As one surgeon explained,

They don't have money for transport and that is the limiting factor. If there is a way they can be supported with funds to return for [follow-up], they will come back. But the limiting factor, even to come for care, the biggest limiting factor is transport.

Another surgeon said succinctly, "The biggest challenge is poverty. Poverty. They don't have the means and they don't have the support from their family. They have been sick, they have not been earning any income."

In addition to these barriers, Ugandan doctors also blamed a lack of perceived value around follow-up care in "Ugandan culture," especially for women who are dry after treatment. As one surgeon explained, "And then there's the culture of follow-up in my society. The culture is-'I feel I'm okay, why should I go [for followup]?' For as long as somebody feels okay, they don't see the need to come to the hospital." Despite these challenges, the surgeons in this study agreed that follow-up care is important because it has the potential to capture critical health indicators, such as residual incontinence, fertility, sexual function, mental health, and quality of life after fistula repair. According to one senior surgeon,

\footnotetext{
We need to follow-up women after fistula repair. It could be three months or six months. The reason is, some of the ladies are discharged when they are dry and they go home. In some cases, you never hear from them again. So surely you cannot know if they are still dry after three, four, or six months. Some of them get pregnant again and we don't even know how they delivered. Did they go hospital, did the fistula recur? So, follow-up is a key component after repair.
}

\section{Tracking Residual Incontinence}

A greater understanding of the scope of residual incontinence can only be achieved with accurate and thorough evaluation and documentation of surgical outcomes, including long-term outcomes. Yet, despite efforts from the $\mathrm{MoH}$ and partners to improve reporting, apparent in the Uganda MoH Fistula Reporting Data Forms (2012a), this information is not available. According to the Ugandan National Obstetric Fistula Strategy (Ministry of Health, 2011),

Currently, there is no standard reliable data system for obstetric fistula whether from facilities or Population based. The lack of accurate data on the prevalence, incidence and 
outcomes of repair regarding obstetric fistula is a challenge to informed decisions on support interventions for policy, planning and programming (p. 18).

Ugandan surgeons, when questioned about the prevalence of residual incontinence, agreed that nuanced tracking of patient outcomes is not standard practice in Uganda despite its obvious benefits. As one surgeon stated, "Well, I couldn't give you figures on that, because we haven't consistently followed that up. We don't have that data, and we wouldn't be routinely documenting that. That information is missing."

Clinical data is collected during fistula treatment camps and at hospitals, then reported to funding agencies, yet these metrics often focus on the numbers of women who received surgery, failing to capture the full range of surgical outcomes. According to one surgeon,

Donors ask, 'How many fistulas did you do last year? How many women did you reach out to? How many operations did you do?' That's what they're interested in. 'Oh, you did 300 last year, okay. We can fund that.'

Another surgeon was even more critical of fistula metrics,

The metrics are fudgeable, they're easily manipulated. I think most of the time the metrics are reported as the number of surgical operations carried out at a particular camp, or over the course of year. People want to see large numbers, with high success rates. So, the success rates are also fudgeable, if you will. Traditionally people simply talked about fistula closure rates that were successful. So, if the fistula was closed, that was a success, even if the urethra was broken and the patient was as wet after surgery as she was before. So, there's not a lot of transparency. There's not a lot of rigor in any of this data. It's really quite soft.

The role of follow-up care in helping to identify and track long-term continence also emerged in interviews with women who left the hospital dry, yet later started leaking, as this woman's story illustrates:

I was expecting to get healed from fistula. And true, the day I was discharged, I was really completely dry. I went home dry. But when I reached home, it was after six months that I started seeing leakage of urine. Every day it became worse, until now it is just like before treatment.

Another woman who had two previous surgeries shared a similar experience:

At my follow-up appointment, they confirmed I still had a fistula. So, I was admitted and they operated on me again. After two weeks I was discharged and I was dry. I went home dry and for the first two days I remained dry. On the third day, I started leaking again.

Despite their current incontinent status, the fact that these women left the hospital dry undoubtedly means that their cases were counted as a "success." Without follow-up care and corresponding tracking mechanisms, their poor outcomes were never reported.

Several doctors offered suggestions to rectify the inconsistencies with, or neglect of, follow-up care, such as additional funds specifically earmarked for follow-up or community-based follow-up care. However, other surgeons were more critical in their assessment, claiming that the lack of follow-up care actually serves donors by 
maintaining higher success rates for fistula surgery than would be reported with improved adherence to follow-up care. According to one surgeon, the paucity of resources and emphasis devoted to follow-up care is directly linked to the fact that "follow-up doesn't appeal to donors." He argued that a careful counting of women with residual incontinence would force donors to acknowledge "partially failed surgeries."

\section{Failure of Policy or Priority? Making Sense of the Neglect of Follow-up}

Women's stories of residual incontinence are stories no one wants to hear as they complicate and make messy the dominant narrative humanitarian organizations and international donor agencies have popularized. Their stories expose the barriers and challenges women face in seeking quality diagnoses and care when they suffer from complications following obstetric fistula surgery. Likewise, surgeons shared their stories and insights into the challenges with tracking residual incontinence and the dynamics that allow poor follow-up to occur. We did not include donor agencies as participants in this study, and as such, we know little about their perspectives on follow-up care nor their funding motivations. Nonetheless, it is clear that a strong commitment to follow-up care is lacking.

What is assumed to matter to international funders are the number of women "successfully" treated. These numbers bolster donors' goals and serve as a cause for celebration on multiple websites where organizations proudly tally the number of women saved from a life of misery (see Heller, 2018). These achievements are then used to raise funds for more surgeries. Fistula funding depends on this narrative of fistula as highly curable (Del Vecchio Good et al., 1990), thus the message of hope is central to building and maintaining funding streams to finance fistula programming. These are complex ethics-international funding for obstetric fistula programs is critical as programs are not financed by national governments nor are they sustainable without continued input from foreign donors. As such, it is easy to empathize with the need to create a compelling story.

Yet, an unintended consequence of this compelling narrative of success is its power to obscure and silence the experience of women who are not cured; women with residual incontinence are systematically omitted from this narrative. Fistula policies do little to counter this narrative. Findings reveal follow-up policies that are vague, absent, and/or unfunded, and thus, not adhered to. Evaluation and revision, essential steps in policy making, and translation of policy into action, all appear to be missing (Buse et al., 2005; Janes \& Corbett, 2009). Results of our study also illuminate the toll failures in clinical assessment of fistula outcomes, data collection, and follow-up care exact on women who are on the receiving end of policies 
that "exact a type of violence of erasure" (Adams, 2016, p. 226). This violence shapes not only their treatment experience, but also determines what problems go unheard, and which potential solutions are left unexplored. This is evident in the dearth of attention, resources, research, and alternative treatment options dedicated to residual incontinence. Women in this study were offered either additional surgery, which has a diminishing likelihood of success with each attempt due to the build-up of scar tissue, or taught to do Kegel exercises, which, given the extreme incontinence many of the women experienced, seemed wildly insufficient. Doctors were also frustrated and discontented with the options available to treat these women.

Ultimately, the solution involves challenging overly simplistic fistula narratives and the reductive representations of obstetric fistula outcomes they promote. Wellfunded fistula policies that seek to provide an accurate and comprehensive account of treatment that also include poor outcomes are needed. Rajkotia (2018) provides insight here, arguing that within the global health community there is intense pressure to report favorable data-so favorable in fact, it may be too good to be true. Surgeons in this study corroborated Rajkotia's argument as they shared their experiences dealing with donors who are predominantly concerned with the number of women treated rather than long-term follow-up or wellbeing. Follow-up care remains an unfunded policy precisely because it has the potential to complicate the narrative of success. Rajkotia also warns that succumbing to the "success cartel" can stifle innovation and progress in global health (2018, p. 1).

Innovation is precisely what is needed to address the pain and suffering associated with residual incontinence. Specifically, there is a need for innovative and context-specific treatment methods that attend to the unique characteristics of residual incontinence post-fistula closure. Both conservative and surgical options need to be explored. Long-term follow-up and accurate assessment of outcomes are critical to these efforts. It is encouraging that several studies have addressed the problem of lack of follow-up, employing not-so-novel strategies such as transportation reimbursement, follow-up via mobile phone, and provision of incentives (Browning \& Menber, 2008; Delamou et al., 2015; Donnelly et al., 2015; Drew et al., 2016; El Ayadi et al., 2019; Maulet et al., 2013; Nielsen et al., 2009; Ouedraogo et al., 2018). For example, Barone and colleagues achieved a follow-up adherence of $96 \%$ at 3 months by offering transportation reimbursement and small appreciation gifts. These studies show that follow-up is both feasible and critical to furthering our understanding of long-term continence, comorbidities, mental health, and social reintegration after fistula treatment. By amplifying women's voices and relaying their experiences to a wider community, our findings provide evidence upon which to build innovative protocols that will improve care for women suffering from fistula and encourage policies at the donor and national level that take into account a long-term, holistic view of health and continence (Barone et al., 2012; Delamou et al., 2016; UN, 2020). 


\section{References}

Adams, V. (2016). Metrics: What counts in global health. Duke University Press. https://doi. org/10.1215/9780822374480

Ahmed, S., \& Holtz, S. A. (2007). Social and economic consequences of obstetric fistula: Life changed forever?. International Journal of Gynecology \& Obstetrics, 99, S10-S15. https://doi. org/10.1016/j.ijgo.2007.06.011

Anastasi, E., Romanzi, L., Ahmed, S., Knuttson, A. T., Ojengbede, O., \& Grant, K. (2017). Ending fistula within a generation: Making the dream a reality. The Lancet Global Health, 5(8), e747e748. http://dx.doi.org/10.1016/S2214-109X(17)30226-7

Arrowsmith, S., Hamlin, E. C., \& Wall, L. L. (1996). Obstructed labor injury complex: Obstetric fistula formation and the multifaceted morbidity of maternal birth trauma in the developing world. Obstetric and gynecological survey, 51(9), 568-574.

Barageine, J. K., Beyeza-Kashesya, J., Byamugisha, J. K., Tumwesigye, N. M., Almroth, L., \& Faxelid, E. (2015). "I am alone and isolated": A qualitative study of experiences of women living with genital fistula in Uganda. BMC Women's Health, 15(1), 73. https://doi.org/10.1186/ s12905-015-0232-z

Barone, M. A., et al. (2012). Determinants of postoperative outcomes of female genital fistula repair surgery. Obstetrics and Gynecology, 120(3), 524. https://doi.org/10.1097/ aog.0b013e31826579e8

Bernard, R. H. (2006). Research methods in anthropology. Altamira Press.

Browning, A. (2006). Risk factors for developing residual urinary incontinence after obstetric fistula repair. BJOG: An International Journal of Obstetrics \& Gynaecology, 113(4), 482-485. https://doi.org/10.1111/j.1471-0528.2006.00875.x

Browning, A. (2012). The problem of continuing urinary incontinence after obstetric vesicovaginal surgery. Obstetric fistula in developing countries. RCOG International News.

Browning, A., \& Menber, B. (2008). Women with obstetric fistula in Ethiopia: A 6-month follow up after surgical treatment. BJOG: An International Journal of Obstetrics \& Gynaecology, 115(12), 1564-1569. https://doi.org/10.1111/j.1471-0528.2008.01900.x

Browning, A., Fentahun, W., \& Goh, J. T. (2007). The impact of surgical treatment on the mental health of women with obstetric fistula. BJOG: An International Journal of Obstetrics \& Gynaecology, 114(11), 1439-1441. https://doi.org/10.1111/j.1471-0528.2007.01419.x

Buse, K., Mays, N., \& Walt, G. (2005). Making health policy (understanding public health). Bell \& Brain Ltd.

Charmaz, K. (2006). Constructing grounded theory: A practical guide through qualitative analysis. Sage Publications Limited.

Creanga, A. A., Iliyasu, Z., \& Arinaitwe, L. K. (2008). An evaluation of the United Nations Population Fund/Uganda's Obstetric fistula program. UNFPA Publication. http://web2.unfpa. org. Accessed 22 July 2018.

Del Vecchio Good, M.-J., Good, B. J., Schaffer, C., \& Lind, S. E. (1990). American oncology and the discourse on hope. Culture, Medicine and Psychiatry, 14(1), 59-79. https://doi.org/10.1007/ BF00046704

Delamou, A., Delvaux, T., Utz, B., Camara, B. S., Beavogui, A. H., Cole, B., et al. (2015). Factors associated with loss to follow-up in women undergoing repair for obstetric fistula in Guinea. Tropical Medicine \& International Health, 20(11), 1454-1461. https://doi.org/10.1111/ tmi. 12584

Delamou, A., Delvaux, T., Beavogui, A. H., et al. (2016). Factors associated with the failure of obstetric fistula repair in Guinea: Implications for practice. Reproductive Health, 13, 135. https://doi.org/10.1186/s12978-016-0248-3

Donnelly, K., Oliveras, E., Tilahun, Y., Belachew, M., \& Asnake, M. (2015). Quality of life of Ethiopian women after fistula repair: Implications on rehabilitation and social reintegration policy and programming. Culture Health \& Sexuality, 17(2), 150-164. https://doi.org/10.108 0/13691058.2014.964320 
Drew, L. B., Wilkinson, J. P., Nundwe, W., Moyo, M., Mataya, R., Mwale, M., et al. (2016). Longterm outcomes for women after obstetric fistula repair in Lilongwe, Malawi: A qualitative study. BMC Pregnancy Childbirth, 16, 2-2. https://doi.org/10.1186/s12884-015-0755-1

ECSA-HC, F. C. E. (2012). The prevention and management of obstetric fistula: A curriculum for nurses and midwives. C. East, and Southern African Health Community/EngenderHealth/ Fistula Care. www.engenderhealth.org/files/pubs/fistula-care-digitalarchive/3/3.1/PreventionManagement-Nursing-Curriculum-English. Accessed 23 March 2018.

El Ayadi, A. M., Barageine, J., Korn, A., Kakaire, O., Turan, J., Obore, S., et al. (2019). Trajectories of women's physical and psychosocial health following obstetric fistula repair in Uganda: A longitudinal study. Tropical Medicine \& International Health, 24(1), 53-64. https://doi. org/10.1111/tmi. 13178

FIGO. (2011). Global competency-based fistula surgery training manual. International Federation of Gynecology and Obstetrics. www.figo.org. Accessed 1 March 2018.

Filippi, V., Ganaba, R., Baggaley, R. F., Marshall, T., Storeng, K. T., Sombié, I., et al. (2007). Health of women after severe obstetric complications in Burkina Faso: A longitudinal study. The Lancet, 370(9595), 1329-1337. https://doi.org/10.1016/s0140-6736(07)61574-8

Fistula Care. (2013). Creating an enabling environment for Fistula prevention and treatment in Uganda. EngenderHealth_USAID. www.fistulacare.org. Accessed 1 Sept 2018.

Gilson, L., \& World Health Organization. (2012). Health policy and systems research: A methodology reader. World Health Organization. https://www.who.int/alliance-hpsr/resources/reader/ en/ Accessed 3 Oct 2018.

Goh, J. T., Browning, A., Berhan, B., \& Chang, A. (2008). Predicting the risk of failure of closure of obstetric fistula and residual urinary incontinence using a classification system. International Urogynecology Journal, 19(12), 1659-1662. https://doi.org/10.1007/s00192-008-0693-9

Hancock, B., \& Collie, M. (2004). Vesico-vaginal Fistula surgery in Uganda. East and Central African Journal of Surgery, 9(2), 32-37.

Harrison, M. S., Mabeya, H., Goldenberg, R. L., \& McClure, E. M. (2015). Urogenital fistula reviewed: A marker of severe maternal morbidity and an indicator of the quality of maternal healthcare delivery. Maternal Health, Neonatology and Perinatology, 1(1), 20. https://doi. org/10.1186/s40748-015-0020-7

Heller, A. (2018). Fistula politics: Birthing injuries and the quest for continence in Niger. Rutgers University Press. https://doi.org/10.2307/j.ctt2111gvj

Janes, C. R., \& Corbett, K. K. (2009). Anthropology and global health. Annual Review of Anthropology, 38, 167-183. https://doi.org/10.1002/9781444395303.ch7

Khisa, W., Wakasiaka, S., McGowan, L., Campbell, M., \& Lavender, T. (2017). Understanding the lived experience of women before and after fistula repair: A qualitative study in Kenya. BJOG: An International Journal of Obstetrics \& Gynaecology, 124(3), 503-510. https://doi. org/10.1111/1471-0528.13902

Koblinsky, M., Chowdhury, M. E., Moran, A., \& Ronsmans, C. (2012). Maternal morbidity and disability and their consequences: Neglected agenda in maternal health. Journal of Health, Population, and Nutrition, 30(2), 124. https://doi.org/10.3329/jhpn.v30i2.11294

Kruk, M. E., Leslie, H. H., Verguet, S., Mbaruku, G. M., Adanu, R. M., \& Langer, A. (2016). Quality of basic maternal care functions in health facilities of five African countries: An analysis of national health system surveys. The Lancet Global Health, 4(11), e845-e855. http:// dx.doi.org/10.1016/S2214-109X(16)30180-2

Lewis, G., \& De Bernis, L. (2006). Obstetric fistula: Guiding principles for clinical management and programme development. Geneva: World Health Organization. https://www.who. int/reproductivehealth/publications/maternal_perinatal_health/9241593679/en. Accessed 3 September 2018.

Maulet, N., Keita, M., \& Macq, J. (2013). Medico-social pathways of obstetric fistula patients in Mali and Niger: An 18-month cohort follow-up. Tropical Medicine \& International Health, 18(5), 524-533. https://doi.org/10.1111/tmi.12086 
Ministry of Health. (2011). National Obstetric Fistula Strategy 2011/2012-2015/2016. Government of Uganda. https://fistulacare.org/archive/files/5/5.4/Uganda_National_Obstetric_Fistula_ Strategy.pdf. Accessed 3 Oct 2018..

Ministry of Health. (2012a). Fistula reporting data forms. Government of Uganda, Department of Clinical Services.

Ministry of Health. (2012b). National training guidelines and standards for treatment of female Genital Fistula. Government of Uganda. https://fistulacare.org/archive/files/8/8.1/8.1.9/ Fistula-training-guidelines.pdf. Accessed 3 Oct 2018.

Ministry of Health. (2014). Minimum Package for conducting Fistula Camps. Government of Uganda.

Morren, G., van den Boogaard, W., \& Dominguez, E. (2016). Management of Obstertic Fistula in Burundi: The experience from a multidisciplinary approach over five years. M.-O. M. Department MSF Project in Gitega Province. Medecins Sans Frontieres. http://www. endfistula.org/sites/default/files/pub-pdf/MSF_Fistula\%20in\%20Burundi_EN-WEB.pdf. Accessed 1 Oct 2018.

Mselle, L. T., Moland, K. M., Evjen-Olsen, B., Mvungi, A., \& Kohi, T. W. (2011). "I am nothing": Experiences of loss among women suffering from severe birth injuries in Tanzania. BMC Womens Health, 11(1), 49. https://doi.org/10.1186/1472-6874-11-49

Murray, C., Goh, J. T., Fynes, M., \& Carey, M. P. (2002). Urinary and faecal incontinence following delayed primary repair of obstetric genital fistula. BJOG: An International Journal of Obstetrics \& Gynaecology, 109(7), 828-832. https://doi.org/10.1111/j.1471-0528.2002.00124.x

Mwini-Nyaledzigbor, P. P., Agana, A. A., \& Pilkington, F. B. (2013). Lived experiences of Ghanaian women with obstetric fistula. Health Care Women International, 34(6), 440-460. https://doi.org/10.1080/07399332.2012.755981

Nielsen, H. S., Lindberg, L., Nygaard, U., Aytenfisu, H., Johnston, O. L., Sorensen, B., et al. (2009). A community-based long-term follow up of women undergoing obstetric fistula repair in rural Ethiopia. BJOG: An International Journal of Obstetrics \& Gynaecology, 116(9), 1258-1264. https://doi.org/10.1111/j.1471-0528.2009.02200.x

Ouedraogo, I., Payne, C., Nardos, R., Adelman, A. J., \& Wall, L. L. (2018). Obstetric fistula in Niger: 6-month postoperative follow-up of 384 patients from the Danja Fistula Center. International Urogynecology Journal, 29(3), 345-351. https://doi.org/10.1007/s00192-017-3375-7

Paxton, A., \& Wardlaw, T. (2011). Are we making progress in maternal mortality? New England Journal of Medicine, 364, 1990-1993. https://doi.org/10.1056/NEJMp1012860. PMID:21612467.

Pope, R., Bangser, M., \& Requejo, J. H. (2011). Restoring dignity: Social reintegration after obstetric fistula repair in Ukerewe, Tanzania. Global Public Health, 6(8), 859-873. https://doi. org/10.1080/17441692.2010.551519

Rajkotia, Y. (2018). Beware of the success cartel: A plea for rational progress in global health. $B M J$ Global Health, 3(6), e001197. https://doi.org/10.1136/bmjgh-2018-001197

Ramsey, K., Iliyasu, Z., \& Idoko, L. (2007). Fistula Fortnight: Innovative partnership brings mass treatment and public awareness towards ending obstetric fistula. International Journal of Gynecology and Obstetrics, 99, 130-136. https://doi.org/10.1016/j.ijgo.2007.06.034

Rane, A., Browning, A., Majinge, P., \& Pope, R. (2020). Challenges in the field of obstetric fistula. International Journal of Gynecology \& Obstetrics, 148, 6-8. https://doi.org/10.1002/ ijgo. 13032

Ruder, B., Cheyney, M., \& Emasu, A. A. (2018). Too long to wait: Obstetric Fistula and the sociopolitical dynamics of the fourth delay in Soroti, Uganda. Qualitative Health Research, 28(5), 721-732. https://doi.org/10.1177/10497323177540

Storeng, K. T., Murray, S. F., Akoum, M. S., Ouattara, F., \& Filippi, V. (2010). Beyond body counts: A qualitative study of lives and loss in Burkina Faso after 'near-miss' obstetric complications. Social Science \& Medicine, 71(10), 1749-1756. https://doi.org/10.1016/j. socscimed.2010.03.056 
Storeng, K., Drabo, S., Ganaba, R., Sundby, J., Calvert, C., \& Filippi, V. (2012). Mortality after near-miss obstetric complications in Burkina Faso: Medical, social and health-care factors. Bulletin of the World Health Organization, 418-425. https://doi.org/10.2471/BLT.11.094011

UBOS. (2018). Uganda Demographic and Health Survey 2016. Uganda Bureau of Statistics and ICF. https://dhsprogram.com/publications/publication-fr333-dhs-final-reports.cfm. Accessed 10 Oct 2018.

UN. (2016). Intensifying efforts to end obstetric fistula. United Nations. https://www.unfpa.org/ resources/intensifying-efforts-endobstetric-fistula. Accessed 10 Oct 2018.

UN. (2018). Intensification of efforts to end obstetric fistula. United Nations. https://digitallibrary. un.org/record/1660278? ln=en. Accessed 4 Jan 2019.

UN. (2020). Intensification of efforts to end obstetric fistula. United Nations. https://digitallibrary. un.org/record/3896019?!n=en. Accessed 11 Nov 2020.

UNFPA. (2008). Campaign to end fistula: The year in review. UNFPA. https://www.unfpa.org/ publications/campaign-end-fistulayear-review. Accessed 7 March 2016.

UNFPA \& EngenderHealth. (2003). Obstetric fistula needs assessment report: Findings from nine African countries. UNFPA.

Wall, L. L., \& Arrowsmith, S. D. (2007). The "continence gap": A critical concept in obstetric fistula repair. International Urogynecology Journal, 18(8), 843-844. https://doi.org/10.1007/ s00192-007-0367-z

Wall, L. L., Karshima, J. A., Kirschner, C., \& Arrowsmith, S. D. (2004). The obstetric vesicovaginal fistula: Characteristics of 899 patients from Jos, Nigeria. American Journal of Obstetrics and Gynecology, 190(4), 1011-1016. https://doi.org/10.1016/j.ajog.2004.02.007

Weston, K., Mutiso, S., Mwangi, J. W., Qureshi, Z., Beard, J., \& Venkat, P. (2011). Depression among women with obstetric fistula in Kenya. International Journal Gynaecology Obstetrics, 115(1), 31-33. https://doi.org/10.1016/j.ijgo.2011.04.015

WHO. (2015). Trends in maternal mortality: 1990-2015: Estimates from WHO, UNICEF, UNFPA, World Bank Group and the United Nations Population Division: Executive summary. https:// www.unfpa.org/publications/trends-maternal-mortality-1990-2015. Accessed 3 Sept 2018.

WHO. (2018). Obstetric Fistula. https://www.who.int/news-room/facts-in-pictures/detail/ 10-facts-on-obstetric-fistula. Accessed 3 Sept 2018.

Wilson, S. M., Sikkema, K. J., Watt, M. H., Masenga, G. G., \& Mosha, M. V. (2016). Psychological symptoms and social functioning following repair of obstetric fistula in a lowincome setting. Maternal and Child Health Journal, 20(5), 941-945. https://doi.org/10.1007/ s10995-016-1950-z

Open Access This chapter is licensed under the terms of the Creative Commons Attribution 4.0 International License (http://creativecommons.org/licenses/by/4.0/), which permits use, sharing, adaptation, distribution and reproduction in any medium or format, as long as you give appropriate credit to the original author(s) and the source, provide a link to the Creative Commons license and indicate if changes were made.

The images or other third party material in this chapter are included in the chapter's Creative Commons license, unless indicated otherwise in a credit line to the material. If material is not included in the chapter's Creative Commons license and your intended use is not permitted by statutory regulation or exceeds the permitted use, you will need to obtain permission directly from the copyright holder.

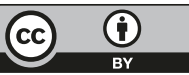

